\title{
PARÂMETROS ARQUITETÔNICO-AMBIENTAIS PARA CONSTRUÇÃO E TESTES EM MODELOS REDUZIDOS, REPRESENTATIVOS DE GALPÕES AVÍCOLAS, COM BASE EM SIMILITUDE
}

\author{
Rolf Jentzsch ${ }^{1}$, Fernando da Costa Baêta ${ }^{2}$, Ilda de Fátima Ferreira Tinôco ${ }^{3}$, Flávio Alves Damasceno ${ }^{4}$, Jairo Alexander \\ Osório Saraz ${ }^{5}$
}

\section{RESUMO}

Considerando a importância do uso de modelos reduzidos no estudo do conforto térmico na criação de aves objetivouse, com o presente trabalho, testar em um galpão comercial de frangos de corte pesadas e três modelos reduzidos construídos em diferentes escalas (1:4, 1:8 e 1:12), a validade das relações propostas e determinar, também, a escala de redução ideal para este tipo de experimento, no qual o conforto térmico foi avaliado por meio do índice de temperatura de globo negro e umidade (ITGU). Com base nos resultados pode-se concluir que, para as condições de operação e critérios de projeto estabelecidos, utilizando o ITGU como índice térmico ambiental de instalações agrícolas, é possível predizer as condições térmicas ambientais em um protótipo, a partir de modelos reduzidos em até 12 vezes.

Palavras-chave: avicultura, conforto térmico, modelos reduzidos.

\begin{abstract}
ARCHITECTURALAND ENVIRONMENTAL PARAMETERS FOR CONSTRUCTION AND TESTING OF REDUCED MODELS FROM TYPICAL POULTRY SHEDS, BASED ON SIMILARITY

Considering the importance of the use of reduced models to the study of thermal comfort on the poultry, this work had the objective to test, in a natural scale of commercial poultry building and three reduced models constructed with different scales (1:4, 1:8 and 1:12), the validity of the relations that have been proposed. Another objective of this work was finding the ideal scale to this type of experiment where the thermal comfort was evaluated using the black globe and humidity (BGHI). Based on results it can be concluded that, for the operating conditions and established design criteria, and using the BGHI as environment thermal index of farms building, it is possible to predict the thermal environment conditions in a prototype based on reduced models up to 12 times.
\end{abstract}

Keywords: poultry, thermal comfort, reduced models

Recebido para publicação em 05/09/2012. Aprovado em 19/10/2012.

1 - Agrônomo, Professor Associado da UFV/Viçosa-MG, jentzsch@ufv.br

2 - Agrônomo, Professor Titular da UFV/Viçosa-MG, baeta@ufv.br

3 - Engenheira Agrícola, Professora Associada da UFV/Viçosa-MG, ftinoco@ufv.br

4 - Engenheiro Agrícola, Professor na UFMT, Campus de Rondonópolis, damascenoufmt@gmail.com

5 - Engenheiro Agrícola, Professor da Universidade Nacional de Colombia, aosorio@unal.edu.co 


\section{INTRODUÇÃO}

O custo elevado envolvido na construção de instalações agrícolas a serem estudadas como, por exemplo, galpões para criação de frangos de corte, muitas vezes inviabiliza a execução de um experimento por falta de recursos (CARVALHO et al., 2009). Neste aspecto, o uso de modelos reduzidos para determinar o desempenho térmico da instalação pode contribuir significativamente para a redução do custo da pesquisa permitindo, ainda, testar várias configurações através da alteração do modelo a um custo mais reduzido que em um protótipo em escala natural.

O uso de modelos é uma ferramenta largamente utilizada na engenharia e requer o entendimento dos princípios que regem a relação entre $o$ modelo e o protótipo. Diversas pesquisas têm sido realizadas com modelos em escala reduzida para solucionar problemas de campo e para facilitar o entendimento de diversos processos físicos, tais como, Murphy (1950), Pattie \& Milne (1966), Neubauer (1972), Silva et al. (1990), Baêta et al. (1996), Moraes et al. (1999), Santos et al. (2005), Evola e Popov (2006), Ferreira Júnior et al. (2009), Norton et al. (2009), Tinôco et al. (2010) e Damasceno et al. (2012). Esses estudos se baseiam na teoria da similitude que tem, por finalidade, estabelecer as relações necessárias para que o comportamento de um protótipo possa ser determinado a partir das observações em um modelo e estabelecer as relações existentes entre as variáveis envolvidas no fenômeno, de forma que os dados possam ser sistematizados com segurança (KÖLTZSCH \& WALDEN, 1990).

A reprodução em escala de fenômenos físicos é vantajosa, por quatro razões: primeiro, quando o problema tratado é muito complexo ou pouco conhecido, sendo necessárias informações empíricas para uma abordagem analítica; segundo, os modelos reduzidos possibilitam a redução do sistema a proporções que facilitam o seu manuseio; terceiro, o uso de modelos permite uma diminuição no tempo gasto na pesquisa e, quarto, proporciona uma maior compreensão do fenômeno investigado.

No geral, considerando que a grande maioria dos trabalhos desenvolvidos na área do conforto térmico para a produção animal envolve o estudo em protótipos ou em modelos reduzidos (DAMASCENO et al., 2012; FERREIRA JÚNIOR et al., 2009; MORAES et al., 1999), este trabalho teve como objetivos: a) determinar, por meio da análise dimensional, as relações necessárias para que o comportamento dos parâmetros de conforto térmico de uma instalação em escala natural possa ser determinado a partir das observações em um modelo reduzido e b) testar em um galpão de criação de frangos de corte e três modelos reduzidos com diferentes escalas (1:4, 1:8 e 1:12), a validade das relações propostas e determinar a escala de redução ideal para este tipo de experimento.

\section{MATERIAL E MÉTODOS}

Visando à determinação da análise dimensional, estabeleceu-se uma lista de variáveis que têm influência no fenômeno estudado. Como o objetivo foi predizer o comportamento térmico no interior de um galpão para frangos de corte, o Índice de temperatura de globo e umidade (ITGU) foi utilizado como indicador do ambiente térmico, sendo influenciado pelas variáveis listadas no Quadro 1.

O Índice de Temperatura do Globo negro e Umidade(ITGU), adimensional, equação 1 engloba, em um único valor, os efeitos da temperatura e da velocidade do ar, da umidade relativa e da radiação (BUFFINGTON et al., 1981).

$\mathrm{ITGU}=\mathrm{t}_{\mathrm{gn}}+0,36 \cdot \mathrm{t}_{\mathrm{po}}-330,08$

em que

$\mathrm{t}_{\mathrm{gn}}$ - temperatura de globo negro $(\mathrm{K})$; e

$\mathrm{t}_{\mathrm{po}}$ - temperatura do ponto de orvalho $(\mathrm{K})$.

$\mathrm{Na}$ determinação das variáveis relativas às dimensões lineares da geometria do galpão, que 
Quadro 1. Variáveis selecionadas para a análise dimensional do comportamento térmico de um galpão para frangos de corte

\begin{tabular}{lllll}
\hline Var. & Grandeza física & Símbolo & Unidade & Dimensão \\
\hline 1 & Largura do galpão & $\mathrm{l}_{\mathrm{g}}$ & $\mathrm{m}$ & $\mathrm{L}$ \\
2 & Dimensões lineares gerais & $\mathrm{d}$ & $\mathrm{m}$ & $\mathrm{L}$ \\
3 & Espessura da telha & $\mathrm{E}$ & $\mathrm{m}$ & $\mathrm{L}$ \\
4 & Absortividade da telha & $\alpha$ & 1 & 1 \\
5 & Condutividade térmica da telha & $\lambda$ & $\mathrm{W} \mathrm{m}^{-1} \mathrm{~K}^{-1}$ & $\mathrm{M} \mathrm{L} \mathrm{T}^{-3} \theta^{-1}$ \\
6 & Irradiância solar & $\mathrm{G}$ & $\mathrm{W} \mathrm{m}^{-2}$ & $\mathrm{M} \mathrm{T}^{-3}$ \\
7 & Velocidade do ar externo & $\mathrm{V}_{\text {ext }}$ & $\mathrm{m} \mathrm{s}^{-1}$ & $\mathrm{~L} \mathrm{~T}^{-1}$ \\
8 & Velocidade do ar interno & $\mathrm{V}_{\text {int }}$ & $\mathrm{m} \mathrm{s}^{-1}$ & $\mathrm{~L} \mathrm{~T}^{-1}$ \\
9 & Índice de temperatura de globo e umidade externo & $\mathrm{ITGU}_{\mathrm{ext}}$ & $\mathrm{K}$ & $\theta$ \\
10 & Índice de temperatura de globo e umidade interno & $\mathrm{ITGU}_{\text {int }}$ & $\mathrm{K}$ & $\theta$ \\
\hline
\end{tabular}

afetam o comportamento do ITGU, considerou-se que a parcela da radiação emitida pelas muretas e paredes das extremidades sobre um termômetro de globo negro localizado no centro do galpão é praticamente nula. Devido às dimensões do mesmo, comprova-se por meio do fator de forma (indica a fração da radiação emitida por uma superfície que é captada por outra), que para um termômetro de globo com diâmetro de $0,15 \mathrm{~m}$, situado a $50 \mathrm{~m}$ e a $5 \mathrm{~m}$ da parede do galpão, será $\mathrm{F}_{\mathrm{ij}}=2 \times 10^{-6} \mathrm{e}_{\mathrm{ij}}=$ $10^{-4}$, respectivamente.

No caso dos modelos reduzidos, o fator de forma para um termômetro de globo negro com diâmetro de $0,06 \mathrm{~m}$, localizado no centro de cada modelo, nas escalas 1:5 e 1:10, para os quais foi considerado o comprimento do galpão real como

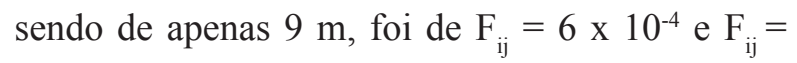
$2 \times 10^{-3}$, respectivamente. Esses fatores de forma foram calculados considerando uma geometria tridimensional e discos paralelos coaxiais (INCROPERA \& DEWITT, 2008).

$\mathrm{Na}$ escolha das variáveis relativas às propriedades termofísicas dos materiais de construção, se destacaram as da cobertura, por representarem $43 \%$ da superfície total do galpão exposta ao ambiente externo. No caso do piso, a influência no comportamento térmico do galpão não foi considerada uma vez que, mesmo em condições de verão, a influência do piso não é significativa por não haver incidência de radiação solar direta sobre o mesmo.

Neste trabalho e de acordo com a teoria da similitude (MURPHY, 1950; KLIN, 1966; KÖLTZSCH \& WALDEN, 1990), em que existe $\mathrm{n}=10$ variáveis e $\mathrm{k}=4$ dimensões básicas envolvidas (massa, M; comprimento, L; tempo, $\mathrm{T}$; e temperatura, $\theta$ ), foram necessários $\mathrm{s}=6$ termos $\pi$ (variáveis adimensionais), listados no Quadro 2.

Quadro 2. Termos $\pi$ adimensionais e independentes

\begin{tabular}{ll}
\hline Número do termo & Termo $\pi$ \\
\hline 1 & $\pi_{1}=\mathrm{d} \cdot 1_{\mathrm{g}}^{-1}$ \\
2 & $\pi_{2}=\mathrm{E} \cdot 1_{\mathrm{g}}^{-1}$ \\
3 & $\pi_{3}=\alpha$ \\
4 & $\pi_{4}=\mathrm{ITGU} \mathrm{ext}_{\mathrm{g}} \cdot \lambda \cdot 1_{\mathrm{g}}^{-1} \cdot \mathrm{G}^{-1}$ \\
5 & $\pi_{5}=\mathrm{V}_{\mathrm{int}} \cdot \mathrm{V}_{\mathrm{ext}}^{-1}$ \\
6 & $\pi_{6}=\mathrm{ITGU}_{\mathrm{int}} \cdot \mathrm{ITGU}_{\text {ext }}^{-1}$ \\
\hline
\end{tabular}


A relação entre as variáveis envolvidas pode ser escrita em função dos termos $\pi$, da seguinte forma:

$$
\mathrm{f}\left(\pi_{1,2} \pi_{2,} \pi_{3,} \pi_{4} \pi_{5}, \pi_{6}\right)=0
$$

Como o termo $\pi_{6}$ contém a variável dependente que se quer predizer $\left(\mathrm{ITGU}_{\text {int }}\right)$, pode-se estabelecer que:

$\pi_{6=} \mathrm{f}\left(\pi_{1,} \pi_{2}, \pi_{3}, \pi_{4}, \pi_{5}\right)$

Pela teoria da similitude, pode-se estabelecer uma relação entre os termos $\pi$ do protótipo e modelo, da seguinte forma:

$$
\frac{\pi_{6}}{\pi_{6 \mathrm{M}}}=\frac{\mathrm{f}\left(\pi_{1}, \pi_{2}, \pi_{3}, \pi_{4}, \pi_{5}\right)}{\mathrm{f}\left(\pi_{1 \mathrm{M}}, \pi_{2 \mathrm{M}}, \pi_{3 \mathrm{M}}, \pi_{4 \mathrm{M}}, \pi_{5 \mathrm{M}}\right)}
$$

Sendo o modelo projetado e operado de mesma forma, tem-se a equação de predição do comportamento do ITGU do galpão:

$\pi_{6 \mathrm{M}}=\pi_{6}$

De acordo com Timmons et al. (1978), os modelos e o protótipo foram expostos ao mesmo fluido (ar) e às mesmas condições do ambiente externo, com relação à irradiância solar, velocidade do ar externo e ao índice de temperatura de globo e umidade externo.

Para garantir que a velocidade do ar externo incidente sobre o protótipo e os modelos fossem iguais $\left(\mathrm{V}_{\text {extM }}=\mathrm{V}_{\text {extP }}\right)$, a velocidade foi medida a uma altura igual à da mureta lateral do galpão $(0,40 \mathrm{~m})$ e os modelos reduzidos foram construídos com a borda superior da mureta a uma altura de $0,40 \mathrm{~m}$ do solo.

A relação entre dimensões lineares $\left(1_{\mathrm{g}}\right)$ homólogas entre os modelos e o protótipo, foi definida pela escala:

$$
\frac{l_{\mathrm{gP}}}{\mathrm{l}_{\mathrm{gM}}}=\mathrm{n}
$$

Esta definição de escala não foi válida para a dimensão linear relativa à espessura da telha, que foi a mesma nos modelos e no protótipo, de modo que EM = EP. A utilização do mesmo tipo de telha nos modelos e no protótipo teve como finalidade a manutenção das propriedades físicas da cobertura em ambos (absortividade e condutividade térmica). A manutenção das propriedades físicas com o uso dos mesmos materiais, segundo Költzsch \& Walden (1990), permite uma similitude maior do comportamento entre os modelos e o protótipo.

Com o estabelecimento das condições de operação, da definição da escala e das propriedades físicas da cobertura, foram determinados os critérios de projeto para a construção dos modelos, apresentados no Quadro 3.

Quadro 3. Critérios de projeto para a construção dos modelos

\begin{tabular}{lll}
\hline Critério & Termo $\pi$ & Condição \\
\hline 1 & $\pi_{1}=\mathrm{d} \cdot 1_{\mathrm{g}}{ }^{-1}$ & $\mathrm{~d}_{\mathrm{M}}=\mathrm{d}_{\mathrm{P}} \cdot \mathrm{n}^{-1}$ \\
2 & $\pi_{2}=\mathrm{E} \cdot 1_{\mathrm{g}}^{-1}$ & $\mathrm{n}=1$ \\
3 & $\pi_{3}=\alpha$ & $\alpha_{\mathrm{M}}=\alpha_{\mathrm{P}}$ \\
4 & $\pi_{4}=\mathrm{ITGU}_{\mathrm{ext}} \cdot \lambda \cdot \mathrm{g}_{\mathrm{g}}{ }^{-1} \cdot \mathrm{G}^{-1}$ & $\mathrm{n}=1$ \\
5 & $\pi_{5}=\mathrm{V}_{\mathrm{int}} \cdot \mathrm{V}_{\mathrm{ext}}^{-1}$ & $\mathrm{~V}_{\mathrm{intM}}=\mathrm{V}_{\text {intP }}$ \\
\hline
\end{tabular}

A condição 1 , referente às dimensões lineares gerais do galpão, determina que os modelos sejam geometricamente similares ao protótipo.

A condição 2, relativa à espessura das telhas, requereu a utilização de telhas com a mesma espessura no protótipo e nos modelos reduzidos, e resultou no uso de modelos distorcidos, tornando necessária a correção da equação de predição.

A condição 3 , referentes à absortividade da telha, determina que a absortividade da cobertura dos modelos e do protótipo seja igual, condição que foi atendida.

A condição 4, refere-se ao uso de modelos em escala natural e resultou no uso de modelos distorcidos, o que requereu a correção da equação de predição. 
A condição 5 , relativa à velocidade do ar no interior do protótipo e dos modelos, determina que estas sejam iguais, o que, em princípio, foi atendido.

Tomando como base modelos em escala reduzida compostos por 3 módulos, calcularam-se os fatores de forma para a troca de radiação entre as empenas e o globo negro com diâmetro de $0,06 \mathrm{~m}$. No Quadro 4 são apresentados os fatores de forma para o protótipo e os modelos reduzidos, calculados para uma geometria tridimensional com discos paralelos coaxiais, considerando-se a empena e o globo como superfícies circulares, situados a uma distância de 1,5 módulos um do outro.

Quadro 4. Fatores de forma das empenas do protótipo e dos modelos reduzidos em relação aos termômetros de globo negro com $0,06 \mathrm{~m}$ de diâmetro

\begin{tabular}{ll}
\hline Tratamentos & $\mathrm{F}_{\mathrm{ij}}$ \\
\hline Protótipo (esc. 1:1) & 0,00001 \\
Modelo 1 (esc. 1:4) & 0,00020 \\
Modelo 2 (esc. 1:8) & 0,00080 \\
Modelo 3 (esc. 1:12) & 0,00176 \\
\hline
\end{tabular}

Os modelos físicos construídos em escalas reduzidas de galpões avícolas foram alocados na área experimental do setor de Melhoramento de Aves do Departamento de Zootecnia da Universidade Federal de Viçosa, nas coordenadas geográficas de $20^{\circ} 45^{\prime} 45^{\prime \prime} \mathrm{S}$ e de $45^{\circ} 52^{\prime} 04^{\prime \prime} \mathrm{W}$, a $651 \mathrm{~m}$ de altitude. Segundo a classificação climática de Köppen, o clima da região é do tipo Cwa, clima quente, temperado chuvoso, com estação seca no inverno e verão quente.

O galpão utilizado como protótipo, com orientação do eixo longitudinal $75^{\circ} \mathrm{NO}$, dimensões de $12,20 \times 147,50 \mathrm{~m}$ e pé direito de $3,00 \mathrm{~m}$. As empenas eram de alvenaria e as laterais possuíam muretas com $0,40 \mathrm{~m}$ de altura e tela com cortina até a cobertura, com abertura da cortina no sentido de baixo para cima. O telhado possuía cobertura de telhas de cimento amianto com inclinação de $33 \%$, beiral com $1,50 \mathrm{~m}$ e lanternim com largura de 2,20 m e abertura vertical de $0,35 \mathrm{~m}$. A estrutura metálica tinha distância entre tesouras de 5,00 m.
Para execução deste estudo, foram construídos três modelos de galpões avícolas em escala reduzida de 1:4 (M1), 1:8 (M2) e 1:12 (M3), cujos comprimentos foram 3,75, 1,88 e 1,25 m, respectivamente. Na Figura 1 são ilustrados os esquemas das seções transversais dos modelos reduzidos M1, M2 e M3.
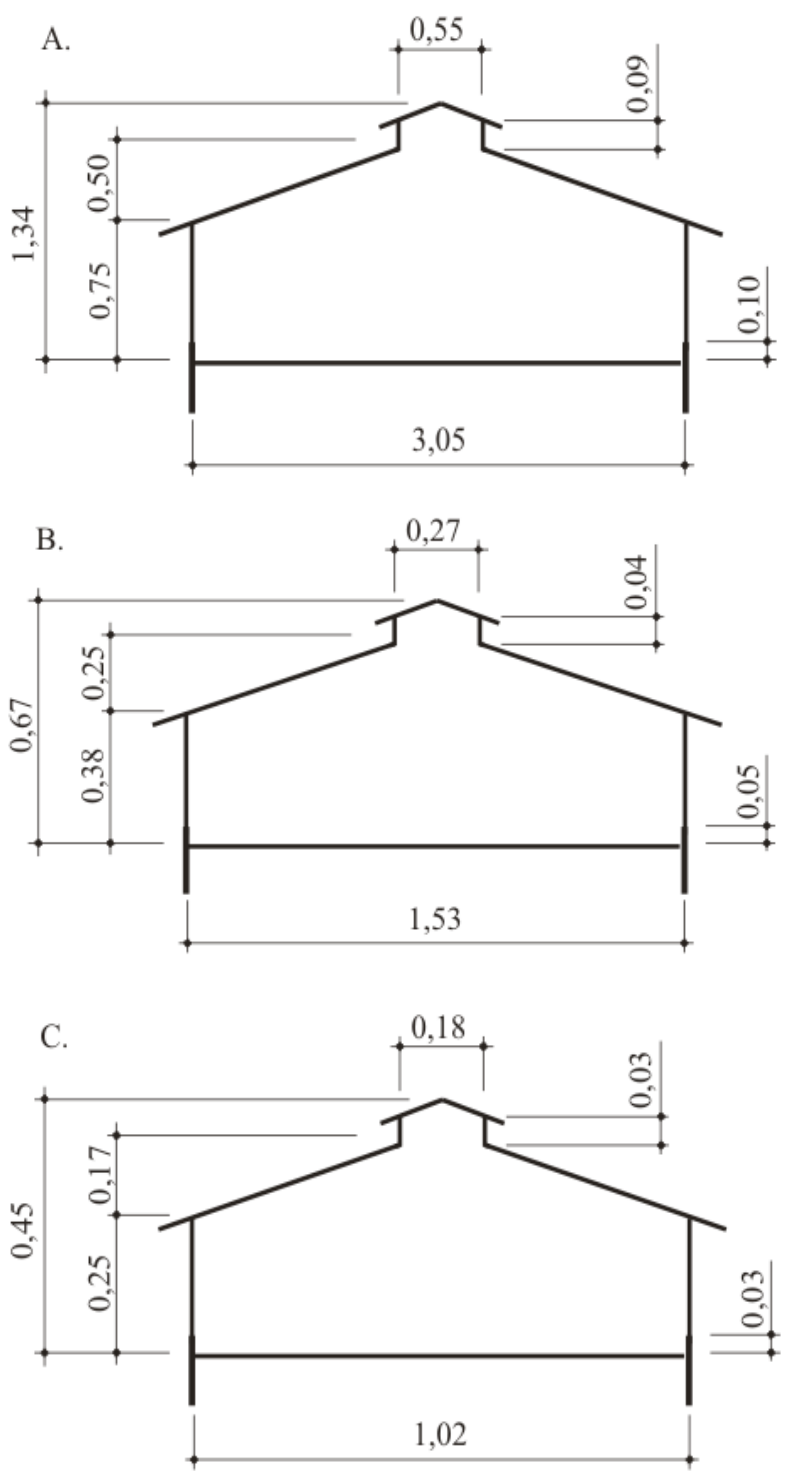

Figura 1. Esquema do corte transversal dos modelos em escalas reduzidos de 1:4 (A), 1:8 (B) e 1:12 (C).

$\mathrm{Na}$ construção dos modelos reduzidos foram utilizadas chapas de compensado $(0,01 \mathrm{~m})$, com estrutura de caibros de madeira maciça, enquanto na cobertura dos três modelos reduzidos foram utilizadas telhas de amianto $(5 \mathrm{~mm})$, apoiadas em 
uma estrutura metálica. Na Figura 2A é ilustrado o posicionamento do protótipo e dos modelos e na Figura 2B uma vista lateral do conjunto.
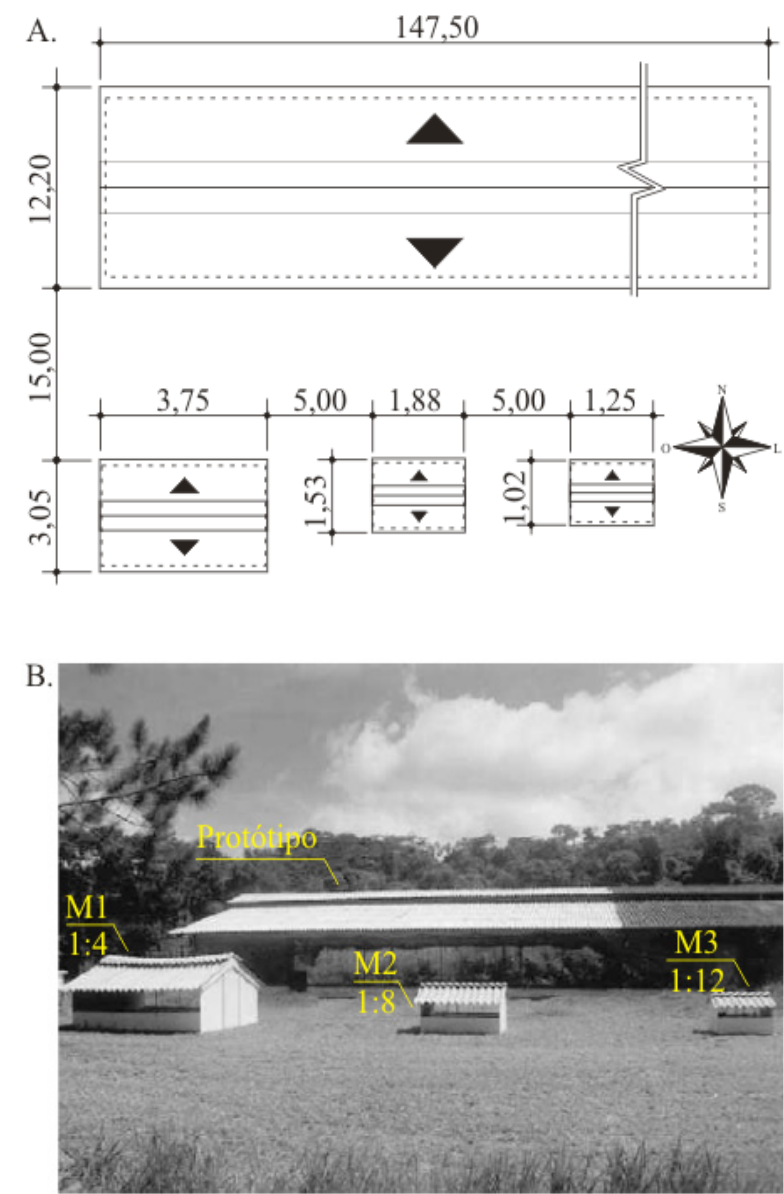

Figura 2. Vista da disposição dos tratamentos (A) em campo e (B) da extremidade do Protótipo e dos modelos reduzidos.

As variáveis de temperatura de bulbo seco $\left(\mathrm{t}_{\mathrm{bs}}\right)$, temperatura de bulbo úmido $\left(\mathrm{t}_{\mathrm{bu}}\right)$ e temperatura de globo negro $\left(\mathrm{t}_{\mathrm{gn}}\right)$ foram medidas no interior dos modelos físicos construídos em escala reduzida e no ambiente externo, durante dez dias, em condição de verão e céu claro.

$A t_{b s}$ e $t_{b u}$ foram registrados a cada minuto, por meio de termopar tipo $\mathrm{T}\left( \pm 0,7^{\circ} \mathrm{C}\right)$. No caso da $\mathrm{t}_{\mathrm{gn}}$, a junção do termopar foi posicionada no interior de um globo de plástico pintado de preto fosco. Os globos utilizados internamente e externamente ao protótipo e modelos, tiveram 0,06 e $0,14 \mathrm{~m}$ de diâmetro, respectivamente. Para a determinação das $\mathrm{V}_{\mathrm{ar}}$ interna e externa aos modelos foram utilizados anemômetros de fio quente $( \pm 2 \%)$ e anemômetros de caneco $( \pm 1 \%)$, respectivamente.

Os sensores de coleta das variáveis ambientais foram instalados no interior do protótipo e modelos na altura correspondente ao centro geométrico das aves. No ambiente externo os dados climáticos foram coletados no interior de um abrigo meteorológico, a $0,40 \mathrm{~m}$ de altura.

A radiação solar foi determinada com o uso de um piranômetro, para radiação solar global $( \pm 5 \%)$, com o registrador programado para armazenar as médias horárias das leituras realizadas em intervalos de $5 \mathrm{~s}$.

$\mathrm{O}$ experimento foi montado segundo esquema de parcelas subdivididas tendo, nas parcelas, os tratamentos (protótipo e modelos reduzidos) e nas subparcelas os horários (1:00 às 24:00), no delineamento em blocos casualizados (DBC), com dez repetições (dias). Os dados foram interpretados por meio de análise de variância processadas pelo software Sisvar 4.6 (FERREIRA, 2000). As médias do fator qualitativo foram comparadas pelo teste de Tukey, a 5\% de probabilidade.

O ambiente térmico também foi analisado de forma descritiva, utilizando-se gráficos Boxplot dos valores observados a fim de caracterizar o ITGU ao longo do período experimental, utilizaram-se, também, medidas de posição e dispersão, como média, mediana e desvio padrão, para complementar as informações obtidas.

A análise de similitude foi realizada de acordo com a metodologia proposta por Timmons et al. (1978) e Murphy (1950), estudando o efeito dos fatores de distorção $\alpha_{\mathrm{k}}$ (a partir de $\pi_{\mathrm{iM}}=\alpha \pi_{\mathrm{i}}$ ) sobre o fator de predição $\delta$ (a partir de $\pi_{1}=\delta \pi_{1 \mathrm{M}}$ ), utilizando para isto os gráficos $\delta-\alpha_{\mathrm{k}}$.

\section{RESULTADOS E DISCUSSÃO}

O resumo das análises de variância para as variáveis respostas $t_{b s}$ e ITGU é mostrado no Quadro 5. Verifica-se que tanto para $t_{b s}$ quanto para o ITGU houve diferença significativa $(\mathrm{p}<0,01)$ para o fator tratamento e horário; apesar disto, nenhuma diferença significativa foi verificada para a interação tratamento $\mathrm{x}$ horário $(\mathrm{p}<0,05)$. Com relação ao ITGU, os coeficientes de variação para as parcelas e subparcelas foram de $0,82 \%$ e $2,22 \%$,

\section{REVENG} 19-30p. 
respectivamente.

Com relação à $t_{b s}$, o teste de médias mostra que não houve diferença significativa entre os resultados nos três modelos reduzidos (Quadro 6), que diferem do Protótipo que, por sua vez, apresentou a menor média, sendo estatisticamente igual ao modelo 3 (teste Tukey, $\mathrm{p}<0,05$ ). Esta diferença pode ser atribuída ao fato da absortividade da telha de cimento amianto do Protótipo ( $\alpha \mathrm{s}$ $=0,49)$ ser menor que a das telhas dos modelos físicos em escalas reduzidas ( $\alpha \mathrm{s}=0,51)$, resultando em uma menor carga térmica devida a radiação solar. Como a cobertura é a maior área exposta do galpão ao meio externo, a diferença de $2 \%$ entre as absortividades pode ter sido determinante nesta diferença de desempenho.

Em relação ao ITGU (Quadro 6), as melhores condições térmicas no interior dos modelos em diferentes escalas reduzidas foram observadas no Modelo 2 e 3, que são estatisticamente iguais (teste Tukey, $\mathrm{p}<0,05)$. Esses resultados confirmam o efeito positivo ao se utilizar modelos reduzidos em escala acima de 1:8.

Pelo comportamento dos valores médios de ITGU nos tratamentos avaliados (Figura 3) podese observar que a maioria dos dados amostrais se concentra dentro da região caracterizada como conforto térmico para as aves, para qualquer tratamento ao longo das horas do dia possibilitando, desta maneira, a comparação, através da análise de

Quadro 5. Análise de variâncias resumida referente ao efeito dos tratamentos e aos horários de observação, em relação a $\mathrm{t}_{\mathrm{bs}}\left({ }^{\circ} \mathrm{C}\right)$ e ao ITGU

\begin{tabular}{llll}
\hline & & \multicolumn{2}{c}{ Quadrados médios } \\
\cline { 3 - 4 } FV & GL & $\mathrm{t}_{\mathrm{bs}}$ & ITGU \\
\hline Bloco & 9 & 73,770 & 143,784 \\
Tratamento & 3 & $0,189^{*}$ & $1,261^{*}$ \\
Resíduo & 27 & 0,022 & 0,348 \\
Hora & 23 & $125,653^{*}$ & $190,848^{*}$ \\
Tratamento x Hora & 69 & $0,129^{\mathrm{ns}}$ & $0,571^{\mathrm{ns}}$ \\
Resíduo & 828 & 1,318 & 2,581 \\
Cv (\%) Parc. & & 0,66 & 0,82 \\
Cv (\%) Subparc. & & 5,03 & 2,22 \\
\hline
\end{tabular}

* significativo a $1 \%$ de probabilidade, ns não significativo.

Quadro 6. Valores médios de tbs $\left({ }^{\circ} \mathrm{C}\right)$ e de ITGU correspondentes aos tratamentos

\begin{tabular}{lllll}
\hline \multirow{2}{*}{ Tratamento } & $\mathrm{t}_{\mathrm{bs}}$ & & ITGU & \\
\cline { 2 - 5 } & Média & & Média & \\
\hline Protótipo & $22,8 \pm 2,26$ & $\mathrm{~b}$ & $72,41+2,34$ & $\mathrm{a} \mathrm{b}$ \\
Modelo 1 & $22,86 \pm 2,24$ & $\mathrm{a}$ & $72,40 \pm 2,22$ & $\mathrm{~A}$ \\
Modelo 2 & $22,87 \pm 2,81$ & $\mathrm{a}$ & $72,38 \pm 2,14$ & $\mathrm{a} \mathrm{b}$ \\
Modelo 3 & $22,84 \pm 2,15$ & $\mathrm{~b}$ & $72,25 \pm 2,06$ & $\mathrm{~B}$ \\
Externo & $23,87 \pm 1,88$ & & $75,54 \pm 5,41$ & \\
\hline
\end{tabular}

Médias seguidas da mesma letra, na coluna, não diferem entre si, pelo teste de Tukey, a 5\% de probabilidade. 
similitude, de uma forma generalizada no interior de toda instalação. Sendo assim, as aves não estariam submetidas a condições de estresse por calor, caracterizado por valores de ITGU superiores a 77 (MEDEIROS et al., 2005).

Para a análise de similitude são apresentados no Quadro 7, os valores das variáveis relativas às características geométricas e das características térmicas das telhas dos quatro tratamentos, utilizadas para o cálculo dos termos $\pi_{1}, \pi_{2}$ e $\pi_{3}$.

No Quadro 8 se encontram as médias gerais dos fatores de distorção e predição sendo que, para o fator de distorção $\alpha_{5}$ e de predição $\delta$, foram determinados os desvios padrão, calculados em função dos valores horários.

$O$ fator de distorção $\alpha_{1}$ depende somente das grandezas geométricas dos tratamentos, ou seja, da razão entre a largura e as demais dimensões geométricas dos modelos em relação à mesma razão do protótipo (Eq. 7).

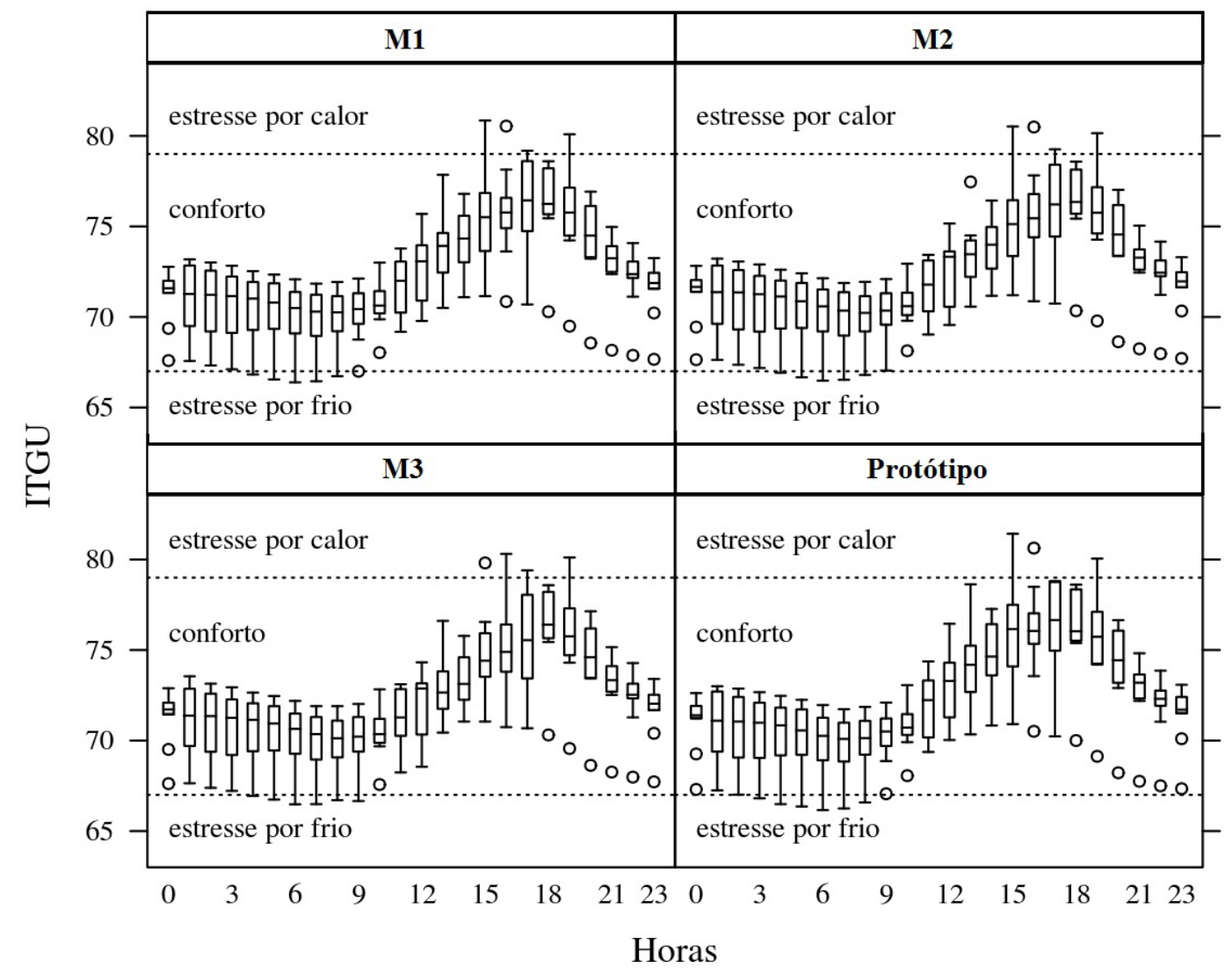

Figura 3. Valores médios de índice de temperatura do globo negro e umidade (ITGU) em função dos tratamentos avaliados e do ambiente externo.

Quadro 7. Variáveis geométricas e físicas dos quatro tratamentos testados no cálculo dos termos $\pi_{1}, \pi_{2} \mathrm{e} \pi_{3}$

\begin{tabular}{lllllll}
\hline \multirow{2}{*}{ Var. } & Grandeza física & Símbolo & $\mathrm{P}$ & $\mathrm{M} 1$ & $\mathrm{M} 2$ & $\mathrm{M} 3$ \\
\hline 1 & Largura do galpão $(\mathrm{m})$ & $\mathrm{l}_{\mathrm{g}}$ & 12,20 & 3,05 & 1,53 & 1,02 \\
2 & Dimensões lineares gerais $(\mathrm{m})$ & $\mathrm{d}$ & 5,00 & 1,25 & 0,63 & 0,42 \\
3 & Espessura da telha $(\mathrm{m})$ & $\mathrm{E}$ & 0,005 & & & \\
4 & Absortividade da telha & $\alpha$ & 0,49 & & 0,51 & \\
5 & Condut. térmica da telha $\left(\mathrm{W} \mathrm{m}^{-1} \mathrm{~K}^{-1}\right)$ & $\lambda$ & 0,58 & & & \\
\hline
\end{tabular}


Quadro 8. Médias gerais dos fatores de distorção $\alpha$ e de predição $\delta$, e desvio padrão (S) do fator de distorção $\alpha_{5}$ e do fator de predição $\delta$

\begin{tabular}{ccccccc}
\hline \multirow{2}{*}{ Fator } & \multicolumn{2}{c}{$\begin{array}{c}\text { Protótipo em relação ao } \\
\text { Modelo 1 }\end{array}$} & \multicolumn{2}{c}{$\begin{array}{c}\text { Protótipo em relação ao } \\
\text { Modelo 2 }\end{array}$} & \multicolumn{2}{c}{ Protótipo em relação ao } \\
\cline { 2 - 7 } & Média & $\mathrm{S}$ & Média & $\mathrm{S}$ & Média & $\mathrm{S}$ \\
\hline$\alpha_{1}$ & 1,0000 & - & 1,0047 & - & 1,0047 & - \\
$\alpha_{2}$ & 4,0000 & - & 7,9739 & - & 11,9608 & - \\
$\alpha_{3}$ & 1,04 & - & 1,04 & - & 1,04 & - \\
$\alpha_{4}$ & 4,0000 & - & 7,9739 & - & 11,9608 & - \\
$\alpha_{5}$ & 2,0244 & 2,9184 & 2,0673 & 2,9891 & 2,0600 & 2,6651 \\
$\Delta$ & 1,0028 & 0,0213 & 1,0030 & 0,0226 & 1,0051 & 0,0267 \\
\hline
\end{tabular}

$\alpha_{1}=\frac{\pi_{1 \mathrm{M}}}{\pi_{1 \mathrm{P}}} \frac{\mathrm{d}_{\mathrm{M}} \cdot \mathrm{l}_{\mathrm{gM}^{-1}}}{\mathrm{~d}_{\mathrm{P}} \cdot \mathrm{l}_{\mathrm{gP}^{-1}}}$

Os valores de $\alpha_{1}=1,0047$ encontrados para o Modelo 2 e Modelo 3, tiveram origem no arredondamento das frações menores que $1 \mathrm{~cm}$ e resultaram em erro menor que $1 \%$, podendo assumir $\alpha_{1} \approx 1$ e que os modelos são geometricamente similares.

$O$ fator de distorção $\alpha_{2}$ depende da razão entre a espessura da telha e a largura dos modelos em relação a mesma razão do protótipo (Eq. 8).

$$
\alpha_{2}=\frac{\pi_{2 \mathrm{M}}}{\pi_{2 \mathrm{P}}} \frac{\mathrm{E}_{\mathrm{M}} \cdot \mathrm{l}_{\mathrm{gM}^{-1}}}{\mathrm{E}_{\mathrm{P}} \cdot \mathrm{l}_{\mathrm{gP}^{-1}}}
$$

A utilização do mesmo tipo de telha no Protótipo e nos modelos teve como finalidade a manutenção das propriedades físicas do telhado e, desta forma, o critério 2 de projeto (Quadro 3 ) não foi atendido e os modelos são geometricamente distorcidos.

Na Figura 4A mostra-se o gráfico de dispersão do efeito do fator de distorção $\alpha_{2}$ em relação fator de predição $\delta$ e a linha de tendência obtida por regressão linear simples $\left(r^{2}=0,83\right)$. Ainda por esta figura, a declividade da linha é praticamente igual a zero, indicando que a distorção provocada pelo o uso de telhas com mesma espessura em todos os tratamentos, afetou pouco o fator de predição $\delta$.

$\delta=0,0003 \alpha+1,0012$
O fator de distorção $\alpha_{3}$ depende da razão entre a absortividade do telhado dos modelos em relação à absortividade do telhado do protótipo (Eq. 10).

$$
\alpha_{3}=\frac{\pi_{3 \mathrm{M}}}{\pi_{3 \mathrm{P}}}=\frac{\alpha_{\mathrm{M}}}{\alpha_{\mathrm{P}}}
$$

Como a absortividade do telhado é a mesma para os três modelos $(\alpha=0,51)$ e ligeiramente maior que a do Protótipo $(\alpha=0,49)$, o fator de distorção $\alpha_{3}$ é igual nos três casos. No gráfico do efeito do fator de distorção $\alpha_{3}$ em relação ao fator de predição $\delta$ (Figura 4B) mostra-se uma linha de tendência vertical, com declividade infinita.

Neste caso, a distorção afeta o fator de predição, uma vez que existe a indicação de elevação de $\delta$ com o aumento da escala n. Como o fator de predição $\delta$ é a razão entre o ITGU interno dos modelos e o do Protótipo (Eq. 11), sendo o ITGU externo o mesmo, o aumento do fator de predição mostra uma tendência de elevação do ITGU interno dos modelos com o aumento da escala n. Esta tendência de elevação do ITGU interno dos modelos pode ser atribuída à relação existente entre a absortividade e a irradiância solar global que, de acordo com as condições de operação estabelecidas, é a mesma para os modelos e o Protótipo $\left(\mathrm{G}_{\mathrm{M}}=\mathrm{G}_{\mathrm{p}}\right)$.

$$
\delta=\frac{\pi_{6 \mathrm{P}}}{\pi_{6 \mathrm{M}}}=\frac{\mathrm{ITGU}_{\text {intP }} \cdot \mathrm{ITGU}_{\mathrm{extP}^{-1}}}{\mathrm{ITGU}_{\text {intM }} \cdot \mathrm{ITGU}_{\mathrm{extM}^{-1}}}=\frac{\mathrm{ITGU}_{\text {intP }}}{\mathrm{ITGU}_{\text {intM }}}
$$


Como em condições de campo não é possível ter a irradiância solar global proporcional às escalas dos modelos, uma forma de neutralizar esta tendência de elevação do ITGU interno em relação ao aumento da escala $\mathrm{n}$ dos modelos, é a introdução de uma distorção intencional, utilizando telhas com absortividade menor nos modelos.

O fator de distorção $\alpha_{4}$ depende da largura do protótipo e modelos, da condutividade térmica da telha e dos fatores ambientais externos, ITGU externo e irradiância solar global (Eq. 12).

$$
\alpha_{4}=\frac{\pi_{4 \mathrm{M}}}{\pi_{4 \mathrm{P}}}=\frac{\mathrm{ITGU}_{\mathrm{extM}} \cdot l_{g M^{-1}} \cdot \mathrm{G}_{\mathrm{M}^{-1}}}{\mathrm{ITGU}_{\mathrm{extP}} \cdot \lambda_{\mathrm{P}} \cdot \mathrm{G}_{\mathrm{P}^{-1}}}
$$

Como os tratamentos foram expostos às mesmas condições de ambiente externo, ITGU $_{\text {extP }}$ $=\mathrm{ITGU}_{\text {extM }}$ e $\mathrm{G}_{\mathrm{P}}=\mathrm{G}_{\mathrm{M}}$, e condutividade térmica das telhas, $\lambda_{\mathrm{P}}=\lambda_{\mathrm{M}}$, o fator de distorção depende apenas das larguras dos tratamentos, apresentando valores iguais aos de $\alpha_{2}$; desta forma, o critério 4 de projeto (Quadro 3) não foi atendido e os modelos são distorcidos. Na Figura 4C mostra-se o gráfico de dispersão do efeito do fator de distorção $\alpha_{4} \mathrm{em}$ relação ao fator de predição $\delta$ e a linha de tendência obtida por regressão linear simples $\left(\mathrm{r}^{2}=0,83\right)$, com declividade praticamente igual a zero, indicando que a distorção afetou pouco o fator de predição $\delta$.

$\delta=0,0003 \alpha+1,0012$

O fator de distorção $\alpha_{5}$ depende da velocidade do ar interno e externo dos tratamentos (Eq. 14).

$$
\alpha_{5}=\frac{\pi_{5 \mathrm{M}}}{\pi_{5 \mathrm{P}}}=\frac{\operatorname{Var}_{\mathrm{intM}} \cdot \operatorname{Var}_{\mathrm{extM}^{-1}}}{\operatorname{Var}_{\text {intP }} \cdot \operatorname{Var}_{\mathrm{extP}^{-1}}}
$$

Como nas condições de operação a velocidade do ar externo é a mesma para os modelos e o protótipo, o fator de distorção $\alpha_{5}$ é a razão entre a $\operatorname{Var}_{\text {intM }}$ e a $\operatorname{Var}_{\text {intP. }}$ Os valores encontrados (Quadro 8) mostram que o critério 5 de projeto (Quadro 3) não foi atendido por nenhum dos modelos e, como mostra o desvio padrão, os dados apresentam uma variação elevada.

$\mathrm{Na}$ Figura 4D apresenta-se o gráfico de dispersão do efeito do fator de distorção $\alpha_{5}$ em relação ao fator de predição $\delta$ e a linha de tendência obtida por regressão linear simples $\left(\mathrm{r}^{2}=0,20\right)$, que apresenta uma declividade pequena, indicando que a distorção afetou pouco o fator de predição $\delta$.

$\delta=0,0253 \alpha+0,9518$

Como o ITGU ${ }_{\text {ext }}$ é igual para todos os tratamentos, o fator de predição é igual à razão

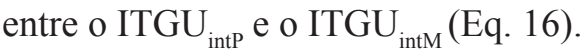

$$
\delta=\frac{\mathrm{ITGU}_{\text {intP }}}{\mathrm{ITGU}_{\text {intM }}}
$$

Sendo assim, a equação de predição (17) do comportamento do Protótipo em relação aos modelos, será:

$$
\mathrm{ITGU}_{\mathrm{intP}}=\mathrm{ITGU}_{\mathrm{intM}} \cdot \delta
$$

Por fim, o fator de predição $\delta$ depende da razão entre o ITGU int $_{\text {e o ITGU }}$ do do protótipo em relação à mesma razão dos modelos (Eq. 18).

$$
\delta=\frac{\pi_{6 \mathrm{P}}}{\pi_{6 \mathrm{M}}}=\frac{\mathrm{ITGU}_{\text {intP }} \cdot \mathrm{ITGU}_{\mathrm{extP}^{-1}}}{\mathrm{ITGU}_{\mathrm{intM}} \cdot \mathrm{ITGU}_{\mathrm{extM}^{-1}}}
$$

Os fatores de predição apresentados no Quadro 8 são valores pouco discrepantes e próximos da unidade, com desvio padrão pequeno, sinalizando um comportamento bastante similar entre os modelos e o Protótipo; esses resultados estão de acordo com os encontrados na análise de estatística, em que a comparação das médias dos valores de ITGU (Quadro 6) mostram que não há diferença estatisticamente significativa entre o Protótipo, o Modelo 1 e o Modelo 2, nem entre o Protótipo, o Modelo 2 e o Modelo 3, pelo teste de Tukey (5\%); desta forma, as distorções existentes nos modelos, devidas ao não atendimento dos critérios de projeto 2, 4 e 5 (Quadro 3), conduzem a um valor para o fator de predição igual à unidade na Eq. 17, que passa a ser: 


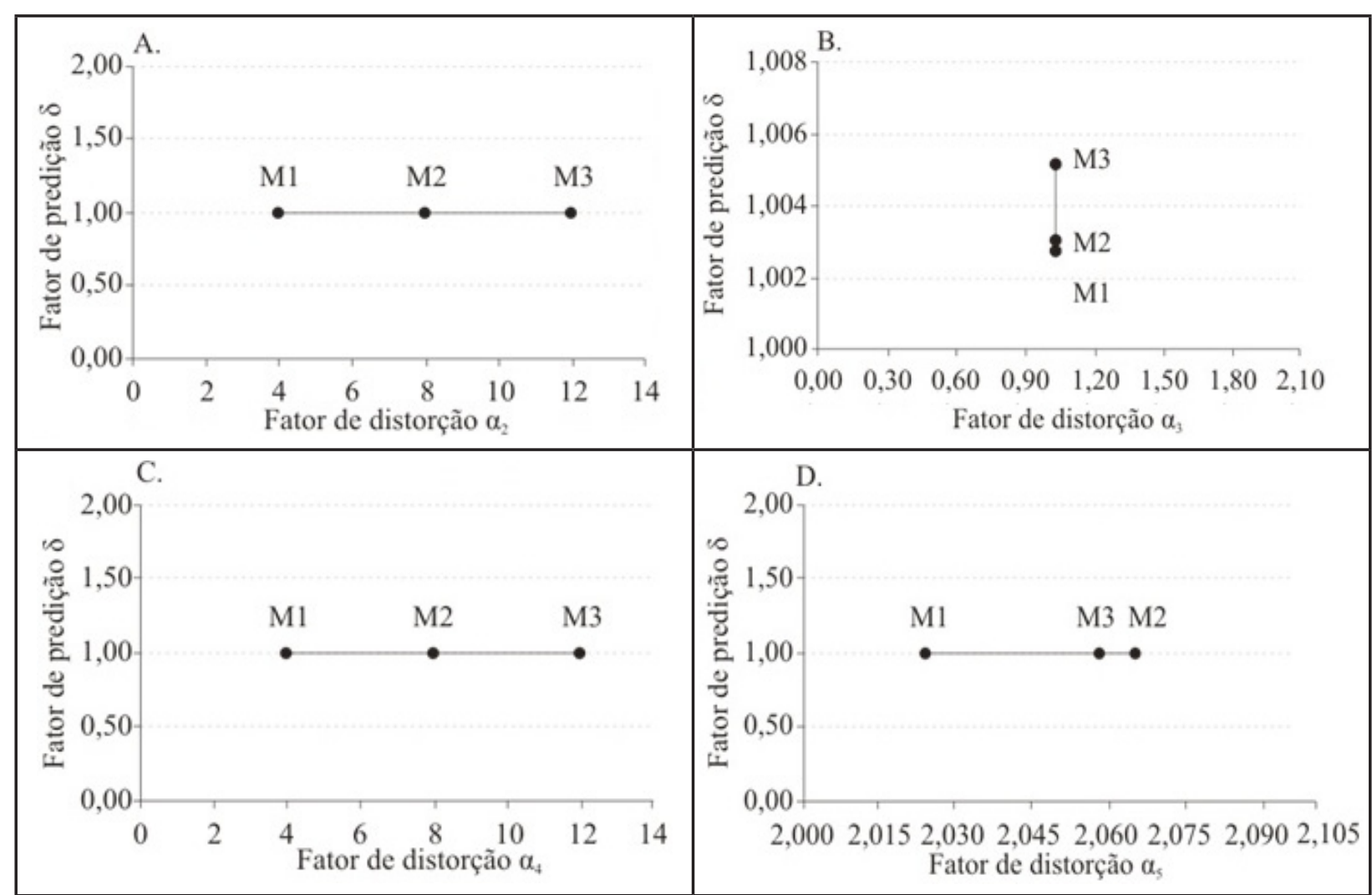

Figura 4. Efeito do fator distorção $\alpha_{2}(\mathrm{~A}), \alpha_{3}(\mathrm{~B}), \alpha_{4}(\mathrm{C})$ e $\alpha_{5}(\mathrm{D})$ sobre fator de predição $\delta$.

$$
\mathrm{ITGU}_{\text {intP }}=\mathrm{ITGU}_{\text {intM }}
$$

Desse modo e como o ITGU é um índice que representa as condições térmicas ambientais do local no qual foram feitas as medições, pode-se concluir que a avaliação térmica ambiental em modelos reduzidos com escala até $1: 12$, pode predizer as condições térmicas do protótipo correspondente.

\section{CONCLUSÕES}

- As distorções existentes nos modelos praticamente não influenciaram $\mathrm{o}$ fator de predição, podendo este ser considerado igual à unidade, para as escala utilizadas;

- O estabelecimento das condições de operação e dos critérios de projeto para o uso de modelos reduzidos, com o emprego de análise dimensional mostrou-se adequado;

- Utilizando-se o índice de temperatura globo negro e umidade (ITGU) como índice térmico ambiental de instalações agrícolas, é possível predizer as condições térmicas ambientais em um protótipo, a partir de modelos reduzidos em até 12 vezes.

\section{AGRADECIMENTOS}

Os autores agradecem ã CAPES, ao CNPq e à FAPEMIG, pelo suporte financeiro.

\section{REFERÊNCIAS BIBLIOGRÁFICAS}

BAÊTA, F.C., DANTAS, F.F., CECON, P.R., PASSOS, E. F. Estudo do termossifão em galpões para frango de corte com cobertura de telhas cerâmicas e de cimento-amianto. Engenharia na Agricultura, v.5, p.1-16, 1996.

BUFFINGTON, D.E., COLLASSO-AROCHO, A., CANTON, G.H., PIT, D. Black globe-humidity index (BGHI) as comfort equation for dairy cows. Transactions of the ASAE. St. Joseph, v.24, p.711-14, 1981.

CARVALHO, V.F., YANAGI JUNIOR, T., FERREIRA, L., DAMASCENO, F.A., SILVA, M.P. Zoneamento do potencial de uso de sistemas de resfriamento evaporativo no sudeste brasileiro. Revista Brasileira de Engenharia Agrícola e Ambiental. Campina Grande, v.13, p.358-366, 2009. 
DAMASCENO, F.A., LOPES, I.M., DAY, G.B., TARABA, J.L., OVERHULTS, D. Study of natural ventilation in reduced model of compost bedded pack barn. In: ASABE Annual International Meeting, 2012, Dallas, Texas. Proceedings... St. Joseph: ASABE, paper $\mathrm{n}^{\circ}$ : 1345189, 2012. CD Rom.

EVOLA, G., POPOV, V. Computational analysis of wind driven natural ventilation in buildings. Energy and Buildings, v.38, p.491-501, 2006.

FERREIRA, D.F. SISVAR - Sistema de análise estatística para dados balanceados. UFLA/DEX, Lavras, Brasil. 2000.

FERREIRA JÚNIOR, L.G., YANAGI JÚNIOR, T., DAMASCENO, F.A.; SILVA, E. , SILVA, G.C.A. Ambiente térmico no interior de modelos físicos de galpões avícolas equipados com câmaras de ventilação natural e artificial. Engenharia Agrícola, v.17, p.166-178, 2009.

INCROPERA, F.P., DEWITT, D.P. Fundamentos de Transferência de Calor e Massa, 6.ed., LTC Editora, Rio de Janeiro, 2008. 567p.

KLIN, S.J. Similitude and approximation theory. New York: McGraw-Hill, 1966, 345p.

KÖLTZSCH,P., WALDEN, F. Ähnlichkeitstheorie und modelltechnik. Heft 1. Freiberg: TU Bergakademie. Freiberg, 1990, 456p.

LEE, I.B., SASE, S., SUNG, S.H. Evaluation of CFD accuracy for the ventilation study of a naturally ventilated broiler house. Japan Agricultural Research Quarterly, v.41, p.53-64, 2007.

MEDEIROS, C.M., BAÊTA, F.C., OLIVEIRA, R.F.M., TINÔCO, I. de F.F., ALBINO, L.F.T.A., CECON, P.R. Efeitos da temperatura, umidade relativa e velocidade do ar em frangos de corte. Engenharia na Agricultura, Viçosa, v.1, p.277286, 2005.

MORAES, S.R.P.M., TINÔCO, I. de F.F., BAÊTA,
F.C., CECON, P.R. Conforto térmico em galpões avícolas, sob coberturas de cimento-amianto e suas diferentes associações. Revista Brasileira de Engenharia Agrícola e Ambiental, Campina Grande, v.3, p.89-92, 1999.

MURPHY, G.C.E. Similitude in engineering. New York: Ronal Press, 1950. 302p,

NEUBAUER, L.W. Optimum alleviation of solar stress on model buildings. Transactions of the ASAE, St. Joseph, v.15, p.129-32, 1972.

NORTON, T., GRANT, J., FALLON, R., SUN, D.W. Assessing the ventilation effectiveness of naturally ventilated livestock buildings under wind dominated conditions using computational fluid dynamics. Biosystems Engineering, v.103, p.7899, 2009.

PATTIE, D.R., MILNE, W.R. Ventilation air-flow patterns by use of models. Transactions of the ASAE, St. Joseph, v.9, p.646-9, 1966.

SANTOS, P.A., YANAGI JUNIOR, T., TEIXEIRA, V. H., FERREIRA, L. Ambiente térmico no interior de modelos de galpões avícolas em escala reduzida com ventilação natural e artificial dos telhados. Engenharia Agrícola, Jaboticabal, v.25, p.575584, 2005.

SILVA, I.J.O, GHELFI FILHO, H., CONSGLIERO, F.R. Materiais de cobertura para instalações animais. Engenharia Rural, Piracicaba, v.1, p.51-60, 1990.

TIMMONS, M.B., ALBRIGHT, L.D., FURRY, R.B. Similitude aspects of predicting building thermal behavior. Transactions of the ASAE, v.21, p.957-962, 1978.

TINÔCO, I.F.F. , SARAZ, J.A.O., PAULA, M.O., PEIXOTO, R.A. F. Elaboración y Evaluation de Placas prefabicadas de concreto aligerado con argilla expandida para uso como coberturas de estructuras pecuarias. Revista Facultad Nacional de Agronomia, v. 63, p. 5465-5744, 2010. 